\title{
A Study on Broad Band Noise Emitted from Underexpanded Radial Jet
}

\author{
Yuichi Hiramoto $^{1 *}$, Hiromasa Suzuki², Masaki Endo ${ }^{3}$, Yoko Sakakibara ${ }^{3}$ \\ ${ }^{1}$ Graduate School of Science and Engineering, Tokyo Denki University, Tokyo, Japan \\ ${ }^{2}$ Tokyo Metropolitan College of Industrial Technology, Tokyo, Japan \\ ${ }^{3}$ Tokyo Denki University, Tokyo, Japan \\ Email: ^17rmt18@ms.dendai.ac.jp
}

How to cite this paper: Hiramoto, Y., Suzuki, H., Endo, M. and Sakakibara, Y. (2019) A Study on Broad Band Noise Emitted from Underexpanded Radial Jet. Open Journal of Fluid Dynamics, 9, 72-81. https://doi.org/10.4236/ojfd.2019.91005

Received: February 10, 2019

Accepted: March 11, 2019

Published: March 14, 2019

Copyright (c) 2019 by author(s) and Scientific Research Publishing Inc. This work is licensed under the Creative Commons Attribution International License (CC BY 4.0).

http://creativecommons.org/licenses/by/4.0/

cc) (i) Open Access

\begin{abstract}
This study focuses on a noise emitted from a radial underexpanded jet. The underexpanded jet is well known as one of supersonic jets and it is formed when the pressure ratio across a convergent nozzle is more than the critical value. The underexpanded jet has been used in many fields, such a propulsion of rocket, laser cutting and a glass tempering process. Such jet is exhausted from a circular nozzle. The expansion wave, the compression wave and the shock wave are periodically formed in the jet, which leads to quasi-periodical shock-cell structure. On the other hand, an underexpanded jet radially issues from intake and exhaust valves of an internal combustion engine and a pressure control valve. The radial underexpanded jet does not have the quasi-periodical shock-cell structure such as the jet from the circular nozzle, its cell length decreasing along jet axis. In this study, an underexpanded jet radially discharged from a circular slit nozzle, which consists of two circular tubes, is experimentally examined for different nozzle pressure ratios. The jet structure is visualized using Schlieren method and a noise emitted from the jet is measured. Typically, the shock associated broadband noise is analyzed and a relation between the jet structure and the radiation noise frequency is discussed. As a result, it is found that the broadband noise lies on lower frequency than a screech tone and that the 1st cell length plays an important role in broadband noise radiation.
\end{abstract}

\section{Keywords}

Broadband Noise, Shock Wave, Underexpanded Jet, Visualization

\section{Introduction}

The underexpanded jet, which issues form a rectangular or a circular nozzle, is 
well known as one of the supersonic jets and it is formed when the pressure ratio across a convergent nozzle is more than the critical value. The underexpanded jet has barrel-shaped cell structure which is quasi-periodical along the jet axis. And it is characterized by the radiation of sounds such as a narrowband screech with a high sound pressure. The quasi-periodical cell structure is responsible for the screech phenomena. Disturbance travels at the jet boundary and interacts with the shock present at each cell node, which leads to radiation of sound wave. The sound wave propagates upstream and interacts with the jet at the nozzle lip, and then new disturbance is originated and goes downstream again. This loop repeats itself and a noise with a narrowband frequency is strongly emitted. This phenomenon has been studied [1]-[6].

In the present study, the underexpanded jet radially spreads out like a disc. Thus, the underexpanded radial jet does not have quasi-periodic cell structure. The underexpanded radial jet is generated inside the pressure reducing valve of the piping and near the poppet valve of the internal combustion engine [7]. In addition, when the underexpanded jet issuing from a nozzle collides with a flat plate at a short distance from the nozzle, the radially spreading wall jet formed on the flat plate also becomes underexpanded [8]. This flow field can be seen when a jet impinges on an object, such as an assist jet during laser cutting or a cooling jet used in a manufacturing process of thermally tempered glass [9]. Thus, understanding the structure of the underexpanded radial jet is very important.

In this study, therefore, a visualization of the jet structure and an acoustic measurement were performed for the underexpanded radial jet. From the results, an influence of the jet structure on acoustic properties of broadband noise was discussed.

\section{Experimental Considerations}

\subsection{Plumbing System}

A schematic view of the experimental apparatus used in this study is shown in Figure 1 . The air compressed by the compressor passes through the air dryer. It is cooled and dehumidified, and then supplied to the surge tank. The pulsating component is removed there. The compressed air steadily flows into the tanks, finally. The pressure in the tank is measured by a digital manometer, and can be adjusted by a valve installed in the pipe. The unnecessary air is discharged to the atmosphere.

In this study, the ratio $P R\left(=p_{0} / p_{a}\right)$ was varied from 2.0 to 4.8 in increments of 0.2 , where $p_{0}$ is the stagnation pressure in the tank and $p_{a}$ the atmospheric pressure.

\subsection{Slit Nozzle}

The air supplied to two tanks goes through a circular pipe fixed on each tank. The circular pipes are arranged facing each other as shown in Figure 2. The cir- 
cular pipe have an inner diameter $d_{0}=14 \mathrm{~mm}$ and an outer diameter $d_{1}=16$ $\mathrm{mm}$. Also, the distance between the ends of the pipes is kept $b_{0}=2 \mathrm{~mm}$. In this study, the nozzle composed of these two circular pipes is called "slit nozzle". The air flow through the pipe accelerates up to the nozzle exit and reaches a sonic speed. The air is underexpanded and radially discharged from the nozzle exit to the atmosphere.

\subsection{Optical Visualization Method}

Shadowgraph method was employed for visualization of jet. Figure 3 shows a schematic view of the visualization system. A light with a flashing time of $180 \mathrm{~ns}$ was used as the light source. The light emitted from the light source is condensed on the pinhole by the condenser lenses. A point light source from the pinhole becomes a parallel ray via a collimator lens, and it is reflected by the mirror " $\mathrm{A}$ " set at $45^{\circ}$ to visualize the jet from the front. The light passing through the jet is reflected by a mirror " $\mathrm{B}$ " set at $45^{\circ}$ and collected by a collimator lens to be photographed with the camera.

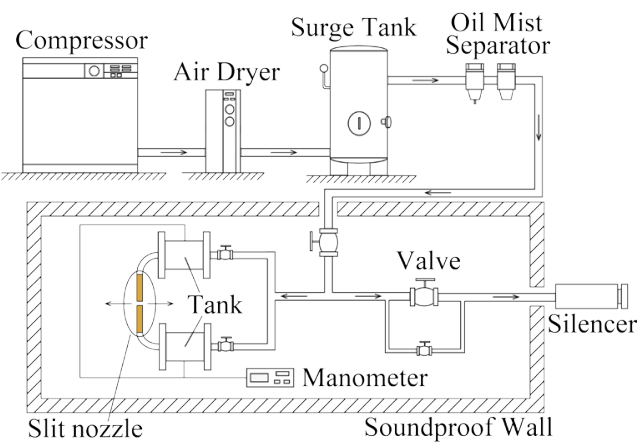

Figure 1. Plumbing system.

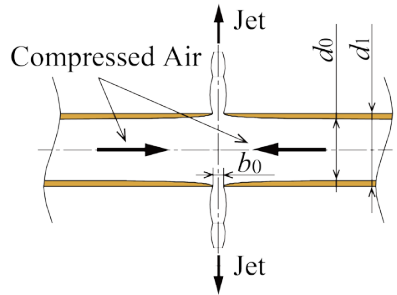

Figure 2. Slit nozzle.

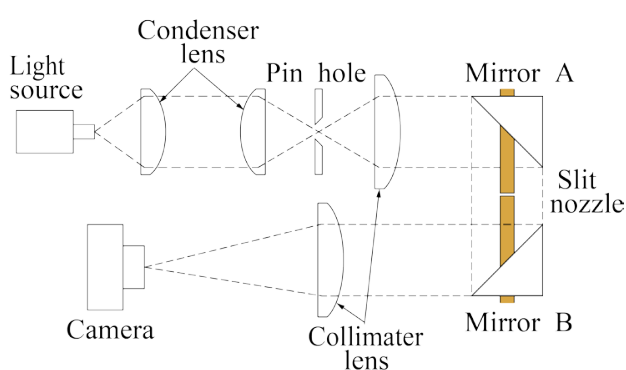

Figure 3. Visualization system. 


\subsection{Acoustic Measurement}

The noise emitted from the jet is measured by the microphone. The sound pressure history of the noise is obtained through an A/D board with $1 \mathrm{MHz}$ sampling. Figure 4 shows an acoustic measurement system. The microphone is attached to the microphone stand at a position separated by $r=750 \mathrm{~mm}$ from the slit nozzle exit. The angle was set at $60^{\circ}$ with respect to the jet axis. In order to prevent reflection of jet noise from the experimental apparatus, a sound absorbing material is glued to the circular tubes comprising the nozzle and the wall surface of the experimental equipment.

\section{Results and Discussion}

\subsection{Visualization Result}

Figure 5 shows jets visualized by shadowgraph method. These pictures are average images of 30 photos randomly taken, respectively. The dark region in the center of the picture is a shadow of the circular pipe and it is larger than the actual diameter due to the optical system. Shock waves cannot be seen when the pressure ratio $P R$ is 2.2, while shock waves surrounding the nozzle can be observed at $P R=2.4$. These annular shocks are called "shock ring". These are referred to as the first shock ring and the second shock ring from the side of the nozzle exit, and it is possible to detect up to the fifth shock ring. At $P R=2.6$ or more, the shock ring becomes unclear on the downstream side and one can find up to the second shock ring. As the pressure ratio $P R$ increases, the distance between the adjacent shock rings increases and the first and the second shock rings become stronger because they can be seen clearly.

\subsection{Cell Length}

Figure 6 shows a photograph taken randomly at $P R=4.8$ and it is not an average image. Since the shock wave ring is closely circular, some points are selected on the shock ring and a circle is specified using a least squares method. The radius of the nozzle is subtracted from the evaluated radius of the first shock ring to obtain the first cell length. In same way, the second cell length was estimated by subtracting the radius of the first shock ring from the radius of the second shock ring. Figure 7 shows the cell lengths. The abscissa is the pressure ratio, and the ordinate the length of the cell. The circle denotes the first cell length, and the square the second cell length. It can be seen that the cell length increases with increase in the pressure ratio as for both the first cell and the second cell. Also, the length of the second cell is always shorter than that of the first cell. This is the features of the radial jet.

\subsection{Acoustic Measurement}

FFT analysis of the sound pressure histories is carried out to find frequency characteristic of the noise radiating from the jet. Figure 8 shows the result obtained 
by FFT analysis. The ordinate represents the sound pressure, and the abscissa the frequency. At every pressure ratio, there is a mountain-type broadband frequency component in the low frequency side and a narrowband frequency component in the high frequency side. A turbulent mixed noise is considered to include in this broadband noise. The narrowband component is screech. The frequency where the sound pressure takes a maximum value at the apex of the mountain shape is measured as $f_{b}$ and the screech as $f_{s}$ as shown in Figure 8(a).

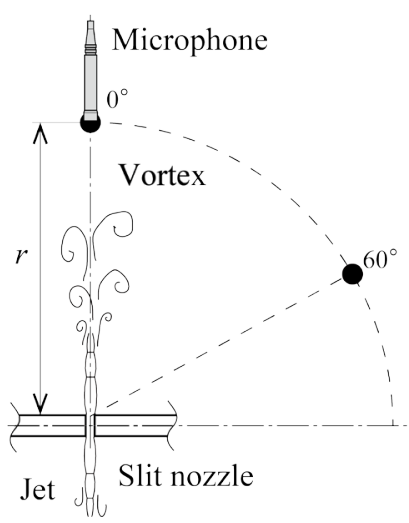

Figure 4. Position of microphone.

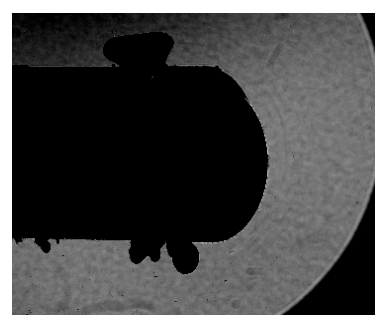

(a) $P R=2.2$

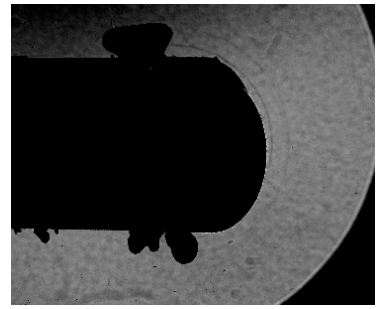

(c) $P R=2.6$

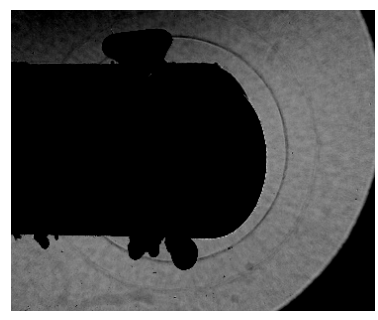

(e) $P R=4.0$

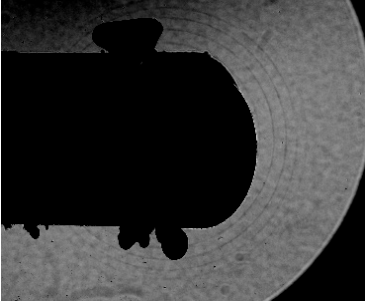

(b) $P R=2.4$

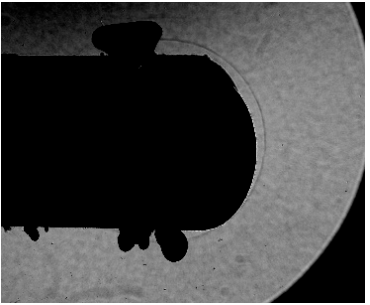

(d) $P R=3.0$

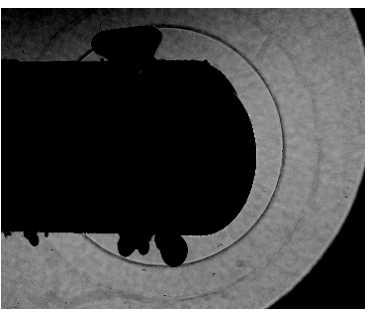

(f) $P R=4.8$

Figure 5. Pictures taken using shadowgraph method. 


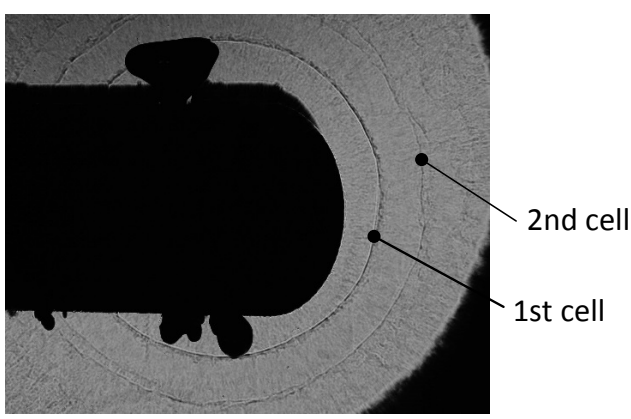

Figure 6. $P R=4.8$ (instant).

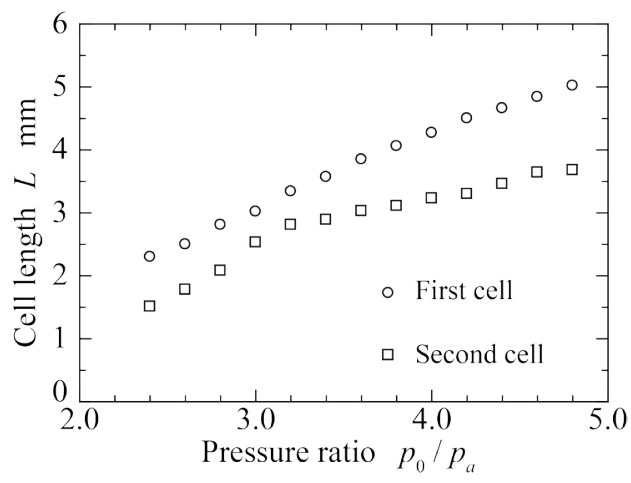

Figure 7. Cell length.

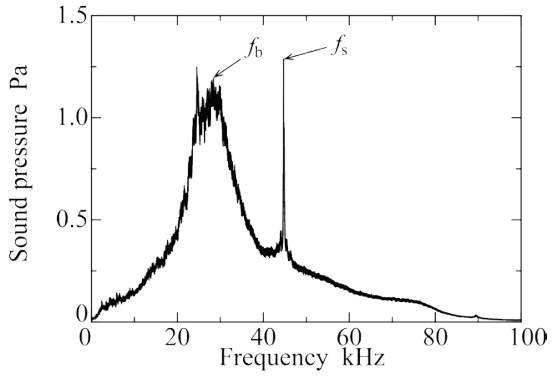

(a) $P R=4.8$

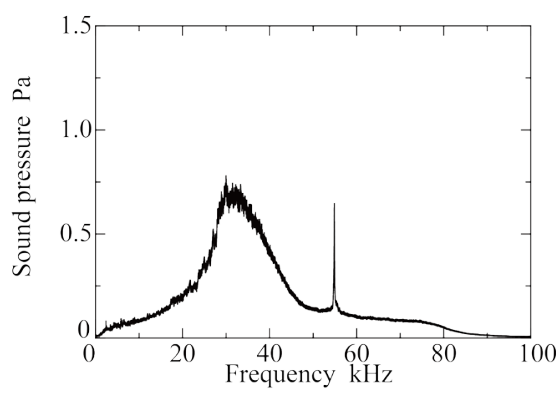

(c) $P R=3.6$

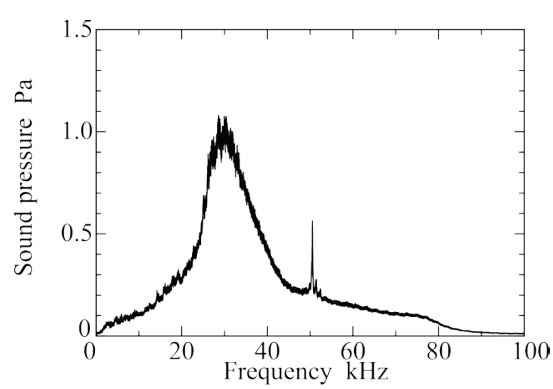

(b) $P R=4.2$

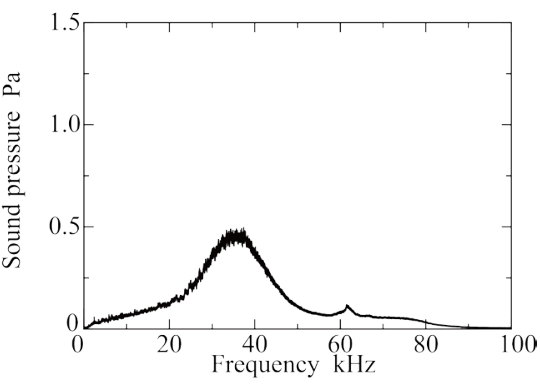

(d) $P R=3.0$

Figure 8. Broadband noise and screech.

Figure 9 shows the measured frequencies $f_{s}$ and $f_{b}$. The ordinate represents frequency and the abscissa pressure ratio. It can be seen that both $f_{b}$ and $f_{s}$ decrease as the pressure ratio rises. $f_{b}$ is found at the pressure ratio of 2.8 or more. 
As shown in Figure 8, along with increase in the pressure ratio rises, both broadband noise and screech develop. Thus, the characteristics of the broadband noise is very similar to those of the screech, this means that the broadband component is caused by the mechanism of feedback loop like the screech phenomena. Kawasaki et al. [10] reported that the single screech frequency was not based on the first nor the second cell but the third cell from the experiment of a radial jet with $d_{1}=16 \mathrm{~mm}$. The second cell length is shorter than the first cell length as shown in Figure 7, and the third one is much shorter. It is expected that the first cell or the second cell contributes to the radiation of the broadband noise if the broadband noise is due to the feedback loop via cell structure.

\subsection{Strouhal Number}

Krothapalli et al. [11] conducted the experiment of underexpanded jet issuing from two types of rectangular nozzle and estimated Strouhal number of screech frequency by the following equation.

$$
S t=\frac{f \cdot D}{U_{0}}
$$

where $f$ is the screech frequency, $D$ the nozzle width, and $U_{0}$ the calculated mean velocity at the nozzle exit for ideally expanded isentropic flow. Krothapalli shows that Strouhal number is approximately obtained by the following empirical equation with high accuracy.

$$
S t=K \cdot P R^{-\frac{3}{2}}
$$

where $P R$ is the pressure ratio, $p_{0} / p_{a}$ and $K$ is the constant value, 0.89 .

In the present study, the Strouhal number can also be obtained by the following Equation (3).

$$
S t=\frac{f \cdot b}{U_{j}}
$$

where $f$ is the frequency, $b$ the cell width, and $U_{j}$ the jet velocity at fully expansion.

Unlike the rectangular jet, the cell width of the radial jet narrows toward the downstream. Let $\bar{b}$ be the average cell width and $\bar{b}$ can be expressed by the following Equation (4) using a continuous equation.

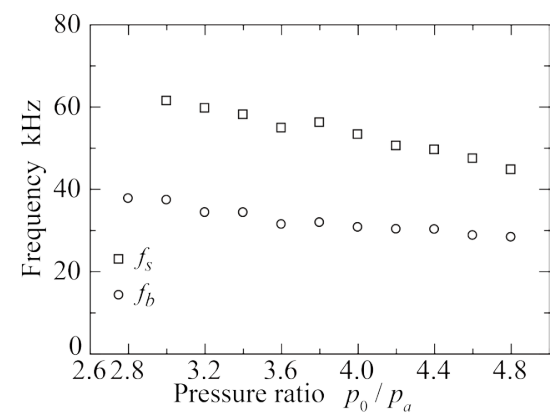

Figure 9. Frequency of noise. 


$$
\bar{b}=\frac{1}{L} \int_{x_{1}}^{x_{2}} \frac{r_{0} b_{0}}{r_{0}+x} d x
$$

where $L$ is the cell length, $r_{0}=d_{1} / 2$ the radius of the nozzle, $b_{0}$ the nozzle exit width. $x_{1}$ and $x_{2}$ are locations of a start and an end of any cell, respectively. In Equation (4), $L_{1}$ is substituted for $L$ and the average first cell width $\bar{b}_{1}$ is calculated, and the average second cell width $\bar{b}_{2}$ is calculated in the same way.

Figure 10 shows Strouhal number of frequency $f_{b}$ calculated with cell width $\bar{b}_{1}$ or $\bar{b}_{2}$. The ordinate is the Strouhal number, and the abscissa the pressure ratio. The red line is the Strouhal number of the screech obtained by the empirical formula, Equation (2), of Krothapalli [11].

The Strouhal number with the cell width $\bar{b}_{1}$ shows good agreement with the empirical formula. From this, it can be considered that the frequency $f_{b}$ is influenced by the screech phenomena related to the first cell rather than that related to the second cell.

\subsection{Coefficient Related to Advection Velocity of Disturbance}

The screech phenomena have been reported to be caused by feedback mechanism due to disturbance [12]. The screech frequency $f$ is given by

$$
\frac{1}{f}=\frac{L}{U_{c}}+\frac{L}{c_{a}}
$$

where $U_{c}$ is the advection velocity of the disturbance, $L$ is the cell length, and $c_{a}$ is the sound speed in the surrounding atmosphere [13]. The advection velocity $U_{c}$ of the disturbance is given by the following equation, Equation (6).

$$
U_{c}=\alpha U_{j}=\alpha M_{j} c_{j}
$$

where $U_{p} M_{j}$ and $c_{j}$ are the jet velocity at fully expansion, the jet Mach number, and the sound speed of jet, respectively. And $\alpha$ is a coefficient for the advection velocity of disturbance and is taken to be $\alpha<1$ from various papers [2] [13] [14] [15].

Assuming that the sound speed at stagnation point in the tank $c_{0}$ is equal to $c_{a}$, Equations (5) and (6) yield the following Equation (7).

$$
\alpha=\sqrt{\frac{\frac{1}{M_{j}^{2}}+\frac{\kappa-1}{2}}{\left(\frac{c_{a}}{f \cdot L}-1\right)^{2}}}
$$

where $\kappa$ is the specific heat ratio, and $\kappa=1.40$ for air. From Equation (6), $\alpha$ was calculated for each pressure ratio with frequency $f_{b}$ and first cell length $L_{1}$. The sound speed $c_{a}$ at the temperature inside the laboratory $\left(20^{\circ} \mathrm{C}\right)$ was used.

Figure 11 shows coefficients related to the advection velocity of the calculated disturbance. The ordinate is the coefficients related to the advection velocity of disturbance, and the abscissa the pressure ratio. The coefficient is 0.37 at $P R=$ 2.8 and it increases gradually and reaches 0.53 at $P R=4.8$. The average value of 


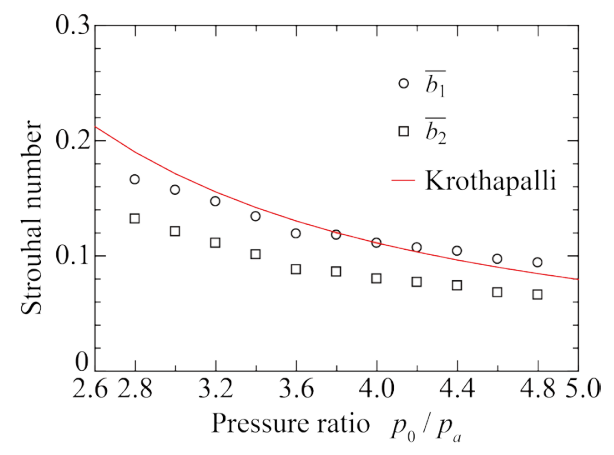

Figure 10. Strouhal number.

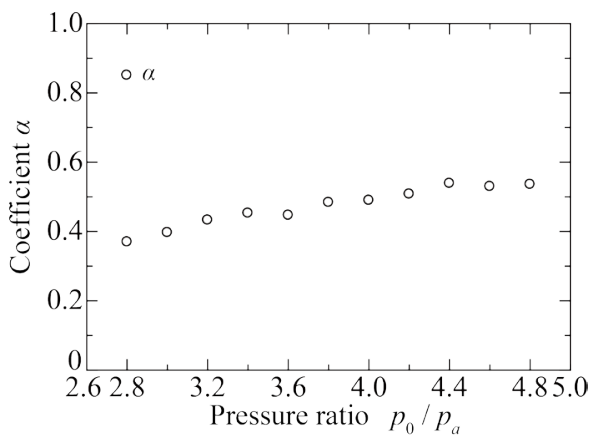

Figure 11. Coefficient for the advection velocity of disturbance.

$\alpha$ was 0.47 . The value is very suitable, compared with the values of $\alpha$ reported so far, i.e. $\alpha=0.42$ by Suda [14] and $\alpha=0.55$ by Raman [13]. From this also, it can be considered that screech phenomena with respect to the first cell length are closely related to the frequency $f_{b}$.

\section{Conclusions}

The underexpanded radial jet is experimentally simulated. The visualization of the jet structure and an acoustic measurement were carried out. From the results, an influence of the jet structure on acoustic properties of broadband noise was discussed and the following conclusions are drawn.

1) The cell length increases as the pressure ratio rises. And it was found that the length of the second cell is shorter than that of the first cell.

2) As the pressure ratio increases, both $f_{b}$ and $f_{S}$ decrease and the broadband noise is developed.

3) The frequency $f_{b}$ is affected by the first cell length and, it is conceivable that screech phenomena of the first cell are closely related to the broadband noise.

It is well known that the jet issuing from a rectangular or a circular nozzle has quasi-periodic cell structure. This is mainly responsible for the screech phenomena. On the other hand, although the radial jet does not have quasi-periodic structure and the cell length becomes shorter along the jet axis, the jet emits the broadband noise and the screech. Therefore, we will need to study what kind of mechanism exists in radiation of the broadband noise and the screech from radial jet without quasi-periodic structure. 


\section{Conflicts of Interest}

The authors declare no conflicts of interest regarding the publication of this paper.

\section{References}

[1] Tam, C.K.W., Seiner, J.M. and Yu, J.C. (1986) Proposed Relationship between Broadband Shock Associated Noise and Screech Tones. Journal of Sound and Vibration, 110, 309-321. https://doi.org/10.1016/S0022-460X(86)80212-7

[2] Tam, C.K.W. (1988) The Shock-Cell Structures and Screech Tone Frequencies of Rectangular and Non-Axisymmetric Supersonic Jets. Journal of Sound and Vibration, 121, 135-147. https://doi.org/10.1016/S0022-460X(88)80066-X

[3] Tam, C.K.W. (1992) Broadband Shock Associated Noise from Supersonic Jets Measured by a Ground Observer. AIAA Journal, 30, 2395-2401. https://doi.org/10.2514/3.11239

[4] Raman, G. (1997) Screech Tones from Rectangular Jets with Spanwise Oblique Shock-Cell Structures. Journal of Fluid Mechanics, 330, 141-168. https://doi.org/10.1017/S0022112096003801

[5] Panda, J. (1998) Shock Oscillation in Underexpanded Screeching Jets. Journal of Fluid Mechanics, 363, 173-198. https://doi.org/10.1017/S0022112098008842

[6] Suzuki, H., Endo, M. and Sakakibara, Y. (2013) A Study on Behaviour of Underexpanded Jet and Induced Vortex. International Journal of Aeroacoustics, 12, 521-538. https://doi.org/10.1260/1475-472X.12.5-6.521

[7] Jibiki, K. (2000) Fluctuation of Steam Control Valve. Turbomachinery, 28, 225-229. (In Japanese)

[8] Carling, J.M. and Hunt, B.L. (1974) The Near Wall Jet of a Normally Impinging, Uniform, Axisymmetric, Supersonic Jet. Journal of Fluid Mechanics, 66, 159-176. https://doi.org/10.1017/S0022112074000127

[9] Aratani, S. and Ojima, N. (1991) Effect of Shock Waves on Fracture and Quenching in Tempered Glass. Proceeding of 18 th International Symposium of Shock Waves, Sendai, 21-26 July 1991, 1277-1282. (In Japanese)

[10] Kawasaki, K., Suzuki, H., Endo, M. and Sakakibara, Y. (2018) A Study on Screech Tone Emitted from Underexpanded Radial Jet. The Seventh Asian Joint Workshop on Thermophysics and Fluid Science, Trivandrum, 21-24 November 2018, 269-274.

[11] Krothapalli, A., Hsia, Y., Baganoff, D. and Karamcheti, K. (1986) The Role of Screech Tones in Mixing of an Underexpanded Rectangular Jet. Journal of Sound and Vibration, 106, 119-143. https://doi.org/10.1016/S0022-460X(86)80177-8

[12] Powell, A. (1953) On the Mechanism of Chocked Jet Noise. Proceedings of the Physical Society, Section B, 66, 1939-1056. https://doi.org/10.1088/0370-1301/66/12/306

[13] Raman, G. (1999) Supersonic Jet Screech: Half-Century from Powell to the Present. Journal of Sound Vibration, 225, 543-571. https://doi.org/10.1006/jsvi.1999.2181

[14] Suda, H., Manning, T.A. and Kaji, S. (1993) Transition of Oscillation Modes of Rectangular Supersonic Jet in Screech. AIAA-93-4323, 15th AIAA Aeroacustics Conference, Long Beach, 25-27 October 1993, 1-9. https://doi.org/10.2514/6.1993-4323

[15] Panda, J., Raman, G. and Zaman, K.B.M.Q. (2004) Underexpanded Screeching Jets from Circular, Rectangular, and Elliptic Nozzles. NASA/TM-2004-212481, 1-12. 